\title{
Influencing Operational Policing Strategy by Predictive Service Analytics
}

\author{
Lisa Jackson \\ Loughborough University \\ L.M.Jackson@lboro.ac.uk
}

\author{
Melanie-Jane Stoneman \\ Loughborough University \\ M.Stoneman@lboro.ac.uk
}

\author{
Heather Callaghan \\ Loughborough University \\ H.A.Callaghan@lboro.ac.uk
}

\author{
Hanjing Zhang \\ Loughborough University \\ H.Zhang@lboro.ac.uk
}

\author{
Christina Latsou \\ Loughborough University \\ C.Latsou@lboro.ac.uk
}

\author{
Sarah Dunnett \\ Loughborough University \\ S.J.Dunnett@lboro.ac.uk
}

\author{
Lei Mao \\ Loughborough University \\ L.Mao@lboro.ac.uk
}

\begin{abstract}
Everyday there are growing pressures to ensure that services are delivered efficiently, with high levels of quality and with acceptability of regulatory standards. For the Police Force, their service requirement is to the public, with the police officer presence being the most visible product of this criminal justice provision. Using historical data from over 10 years of operation, this research demonstrates the benefits of using data mining methods for knowledge discovery in regards to the crime and incident related elements which impact on the Police Force service provision. In the UK, a Force operates over a designated region (macro-level), which is further subdivided into Beats (micro-level). This research also demonstrates differences between the outputs of microlevel and macro-level analytics, where the lower level analysis enables adaptation of the operational Policing strategy. The evidence base provided through the analysis supports decisions regarding further investigations into the capability of flexible neighbourhood policing practices; alongside wider operations i.e. optimal officer training times.
\end{abstract}

\section{Introduction}

Analytics, a multidisciplinary field that finds application in various sectors, has traditionally been used to inform decision-making. The use of analytics is ever increasing being the key factor in enhancing real time decision-making capabilities. This systematic computational analysis of data/ statistics, i.e. analytics, focuses on the analysis of large amounts of data, applying robust data analysis techniques [1]. The data analytics that became popular in the early 2000s, largely due to the development in technology, presents high interest in the developing world since it enables the enhancement of productivity and business gain offering high levels of service quality [2, 3]. This evolving role of analytics features in a wide spectrum of sectors such as in public services, healthcare and pharmaceutical industry, financial markets and institutions, media companies, mining and resources, energy and other disciplines.

Moreover, the data analytics evolution is well underway in the healthcare sector and pharmaceutical industry and is able to help patients, doctors, physicians and other relevant stakeholders make better use of available healthcare information. Analytics is used in several ways in healthcare sector. For instance, using health information, analytics can provide a more generic picture of health issues providing a better understanding of which treatments deliver the best or less effective outcomes. This can help both doctors make informed decisions but also pharmaceutical companies improve their market intelligence. Additionally, predictive analytic methods have been used to detect patients who belong to high risk populations, i.e. individuals/ groups with conditions such as HIV, diabetes, hypertension and other diseases who are at high risk of non-adherence to medications. Also, early alerts on diseases may be detected and prevented, with the pharmaceutical industry to be able to respond in real time [4].

Analytics is also used in the financial services sector and focuses on the prediction of needs, issues and behaviour of clients in order to provide a more personalised customer experience, identify the right markets and customers, and make the right strategic decisions. Meanwhile, analytics is being increasingly used in the media industry allowing a better understanding of the needs and preferences of the audience. Once the media companies detect the customer preferences and identify new products/ services relevant to these preferences, they increase the relevance of their communications personalizing the offerings by deploying intelligence to the customer website and mobile applications [5].

Hence, the use of analytics can lead to safe and informed choices since the decisions and strategies are based on historical data and the effectiveness of a 
strategy is determined in real time. Thus, with powerful insights, analytics could likely reduce costs of businesses and organisations and optimise their operational efficiency increasing their profits and saving time and effort.

The Police Force is increasingly turning to predictive policing, i.e. the application of analytics in the fight against crime; to respond to crime by developing appropriate strategies, to predict potential targets so as to prevent criminal acts, and to make police investigations more productive. Predictive policing begins with the analysis of historical crime data and applying data mining methods. Using predictive analytics the Police Force is able to forecast places and times where there is an increased risk of crime. There has been much research on using crime data to assist with the positioning of police resources. Malik et al. [6] used novel spatial and temporal analytical techniques, as well as a novel dynamic covariance Kernel Density Estimation (KDE) method, in a comprehensive study, to assist with resource allocation. Other authors have used evolutionary optimisation methods, alongside historical crime data analysis and KDE, to position officers [7]. The use of a sketch-based approach has been proposed for dynamic route planning by Godwin et al. [8], which allows a police officer to rapidly specify a path through the city without typing and reviewing the volume and types of crime that occur along the route. Analytics have also been used to identify potential individuals at risk of offending in the future, predict likely victims of crimes or predict perpetrators' identities, creating profiles that match potential offenders with specific past crimes [9, 10]. A framework for application and relationships of crime data mining techniques has been developed by $[11,12]$ and commercial software for the analysis and prediction of criminal activity is available and used in some forces (predominantly in the U.S, Predpol). Therefore it is evident that data-driven analytics and insights can play a fundamental role in acquisition of crime knowledge, improving the quality and operational efficiency in the delivery of Police Force services with the ultimate goal: the optimisation of their provision services performance.

The UK Police Force have for some time collected data about crimes and related incidents. Having an analytics based solution that works in the practical Police environment in the UK though is still not completely resolved. With a move towards predictive policing, making decisions supported by an evidence base, there is a desire to use the capability of techniques for analyzing large quantities of data to glean new insights from such analysis, to move away from subjective decisions. Though most Forces do have analytics teams, they are nascent in using analytics in a predictive/preemptive way, rather than reactively which is more common place, due to a lack of resources.

The contribution of this paper is in knowledge elicitation of criminal activity and incident occurrence from a proactive point of view, spanning a large time frame, which is currently not in existence. The authors are not aware of any research in the literature that focuses on knowledge acquisition gained through using analytics regarding micro and macro level differences and their implications as shown in this paper. Especially in the UK with future planned changes i.e. boundary-less policing, these differences could have potential implications for resource allocation strategies, amongst other things.

Despite, at this stage, utilizing just basic analytics, there has been a leap forward in knowledge discovered from such research. The types of insights that are likely to emerge from criminal analytics and the process (and infrastructure - analytic algorithms) adopted to elicit these insights are discussed. Our findings have policy implications regarding operational activities and as such have supported the motion to enhance the level and depth of analytics, encompassing a wider range of data sources, to investigate such operational implications.

\section{Police operation and infrastructure}

\subsection{Regions and Beats}

In any country with a developed code of law there is the need to employ some form of policing body to ensure its upkeep and to maintain a standard of order and public safety. To this end the United Kingdom nationally employs a total of more than 160,000 officers across the entire standard policing structure. This figure is broken down into a variety of necessary officer roles; this is then further spread across all the policing regions of the UK. There are 43 police forces across England and Wales, broken down to cover a specified geographical area governed by known boundaries typically following County boundaries. Each force operates a regional service, where its region is further subdivided. Neighbourhood Policing Teams (NPT) involves smaller teams of police officers who are dedicated to policing a certain community or area, referred to as a Beat. Typically allocation of resources, operational activity and statistics is carried out at Force level as opposed to Beat level. 


\subsection{Roles and Activities}

Through suitable allocation of the resources Forces seek to provide the best service possible to the public whilst simultaneously maintaining the requirements laid out in the Quality of Service Commitment. This document details 50 key criteria that must be fulfilled by any regional policing body. Additionally each Police and Crime Commissioner have their own Police and Crime plans, which identify localised priorities which enforces further guidelines on Police activity when interacting with members of the public.

Thus a major problem that the Police Force find themselves confronted with is how to spend the resources they have available in order to best meet all of the demands and constraints made upon the services they provide.

This demand comes in a number of forms and originates from a variety of sources. Not only do Police officers respond to calls from the general public but they are also constantly undertaking initiatives in order to aid in the prevention and detection of crime. For example major public and sporting events will usually have continued attendance of a number of dedicated officers in addition to generating a sizeable amount of office/paper work. The primary focus of this research is to understand the crime and incident portfolio through the use of analytics. This is facilitated by the use of large historical data sets that are currently not used for this purpose. The focus is to look at using the data proactively, as opposed to reactively, focussed on initial problem solutions in the current time frame, which is predominantly where the majority of analysis occurs, typically due to resource limitations.

\section{Current Data}

The data available relates to crimes and incidents collected on designated Police databases. For the crimes historical records from 2006 onwards have been used in the research. The crime types presented in the research include 5 types: i) Assault with violence; ii) Assault without violence; iii) Criminal damage; vi) Vehicle offences; and v) Theft/Burglary. The dataset providing information on crimes has the following fields:

- Occurrence number

- Reported date of crime

- Start date of crime

- Start time of crime

- End date of crime

- End time of crime
- Whether the crime was related to Domestic Violence

- Whether the crime was related to hate crime

- Type of crime - Home Office descriptions

- Location data $-\mathrm{x}$ and $\mathrm{y}$ co-ordinates.

Data has also been provided on incidents. Incidents are issues that are reported to the police and include events such as public safety, anti-social behaviour, crime and transport. The data provided covers the period of April 2007 to January 2017. The dataset has the following fields:

- Reference number

- Incident date

- Incident time

- Incident grade

- Incident result (Category)

- Location data (eastings and northings)

Data within this information falls into six main categories:

- Anti-Social Behaviour (ASB) - including environmental, neighbour related or personal

- Crime - This does not include all recorded crimes, only those reported to the police

- Admin Incidents - Used mainly for messages, contact records, police generated resource activities and complaints against the police.

- Public Safety - This includes incidents where the police have been contacted as there is a concern for safety, suspicious circumstances, vulnerable person, domestic incidents (where no crime involved), missing persons etc.

- Qualifier - This is an old category no longer used, however most of the records relate to ASB.

- Transport - This includes road related disruption, road traffic accidents and aircraft/rail/marine incidents.

- Miscellaneous - used for historical / testing purposes

The different start dates for the data is due to the Force collection process and hence there has been no merging of the data, the crime/incidents are analysed separately. For all the data it can be broken down into local policing areas (NPA) and neighbourhoods / beats (NPT). In terms of the NPA there are a total of 79,287 crime data records and 253,373 incident records. The NPT referred to in this paper is a primarily urban, residential area with a single main street comprised of small commercial businesses, many of which are independently owned. These enterprises include 
convenience stores, fast food takeaways and small retail shops. Also included in this area are a sports stadium and part of a university. Specifically, 14,708 and 44,385 of the total crime and incident records respectively, relate to this beat.

\section{Preliminary Analysis}

\subsection{Preview of Fundamental Analysis}

The analytic methodology involved acquisition of business understanding, understanding the two databases, preparation of the data, the analysis itself and the evaluation of it. The analysis steps adopted in the research are outlined in Figure 1. Initial focus was a trend analysis, followed by the use of clustering methods, where a breakdown was carried out for differing time frames and segregation of crime and incidents into their various subdivisions. Both of these methods were employed at the macro and micro levels. The outputs have led to knowledge discovery regarding elements influencing policing service provision and have provided the impetus for application of more advanced analytics in key areas.

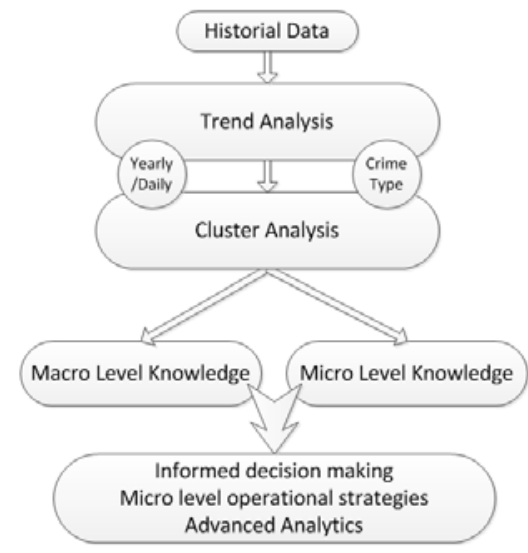

Figure 1. Flow chart of process

\subsection{Data cleaning}

Initial cleaning of the data was required. The cleaning entailed removal of: historic crimes (i.e. sex crimes that did not occur in the time period); non-recordable crimes; records with a start date that falls outside of the key period starting $1 / 1 / 2006$; and records where there was no start date or end date or both. For the incident data, excluded from analysis were: records that had a main category of crime incidents (i.e. a duplication of a crime record), records where no type of incident was recorded, and records that had a main category of Miscellaneous. In addition, some recoding needed to occur due to changes in recording format. After cleansing the population records totalled: NPA crime data 78,916; micro (beat) crime data 14,641 records. The total population incident data reduced to 187,015 records, with the beat incident data having 32,552 records.

\subsection{Trend analysis}

\subsubsection{Method}

The aim of the trend analysis was to give some context to the detailed analysis to follow. Annual trend was investigated by plotting the yearly number of crimes and incidents for the Beat. This was based on either the start date of the crime or the reported date for incidents. To get an idea of how the beat trend compared to the policing area, a separate chart was created, plotting the annual number of crimes / incidents for the whole policing area, alongside the proportion of crimes that occurred in the beat. This enables us to see whether an increase in beat crimes was due to an increase in overall crime for the area, or whether it was just at beat level. In addition to the overall trend, the data can be analyzed to look at trends in day of the week or time of day. This trend analysis can then be repeated for crime / incident type to provide more detail and context setting.

\subsubsection{Results}

Initially reviewing the historical trend with regards to crime and incidents at NPT level, the number of reported incidents in the beat is over double the number of reported crimes (shown in Figure 2). The start date for comparison is 2007 due to no records for incidents in 2006. Both crimes and incidents experienced a decrease between 2008 and 2103, however 2104 saw an increase in incidents reported. Both crimes and incidents increased in 2016.

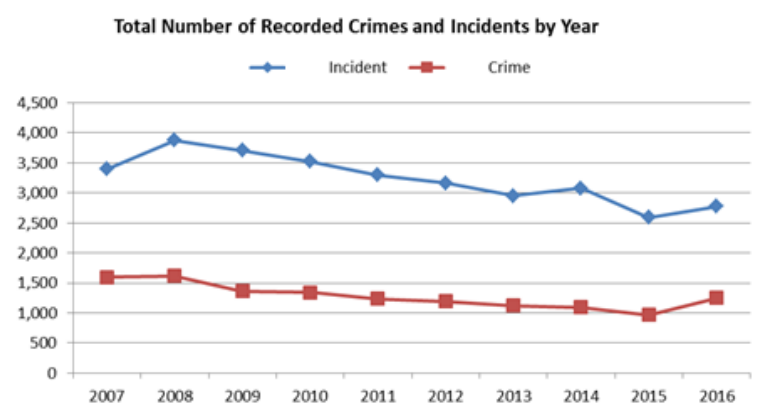

Figure 2. Beat level crime and incident trend 
Over the period, crimes in the beat accounted for around $19 \%$ of recorded crimes in the policing area, even though the total number of recorded crimes decreased between 2006 and 2014 (Figure 3). Interestingly, when recorded crimes for the policing area increased in 2015, the proportion of crimes occuring in the beat decreased, suggesting this increase was not due to an increase in the beat.

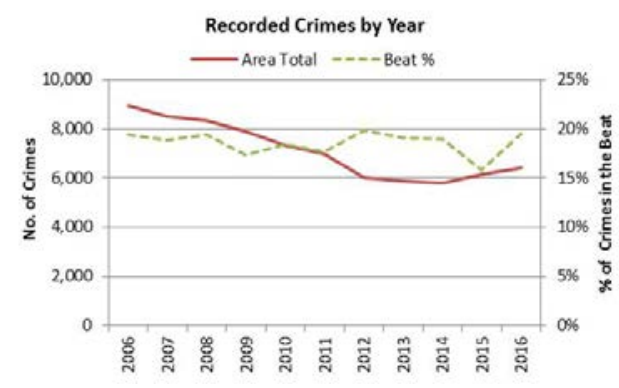

Figure 3. Beat/Region comparison

Although over the whole period the beat accounted for $19 \%$ of recorded crimes across the policing area, this was not the same across the different crime types. For example, only $10 \%$ of domestic burglaries in the policing area happened within the beat. However 23\% of assaults did occur within the beat. This has implications when setting priorities at an area level rather than a beat level.

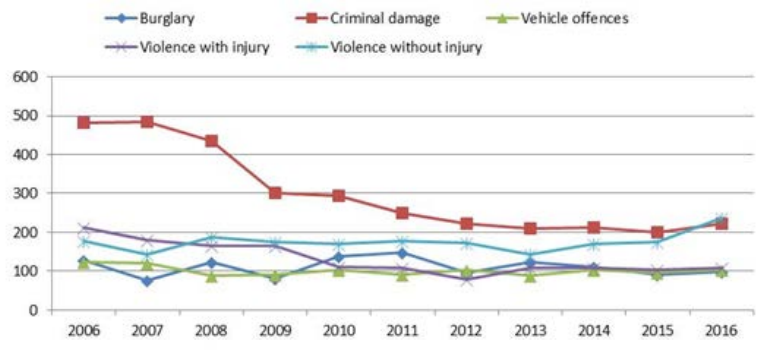

Figure 4. Beat crime type trend

Figure 4 shows trend data for five of the main offence groups in the beat. The crime groups are within the Home Office Crime Tree Mapping framework and are those identified by the Force as their priority areas. Over the period, nearly a quarter of crimes recorded in the beat were criminal damage offences. There was a relatively large decrease in these offences between 2008 and 2009 which warrants further investigation. Although violence against the person offences (with and without injury) accounted for a similar proportion as criminal damage, there have been changes in trend over the last ten years. The number of violence with injury offences has halved when comapring 2006 to
2016. Conversley, the number of violence without injury offences remained stable, apart from in 2016, which saw a 35\% increase on the previous year.

As with the trend for the beat, the number of incidents recorded for the policing area decreased between 2008 and 2015, with a slight increase in 2016 (Figure 5). However, the proportion of incidents that occurred within the beat also decreased from 19\% in 2008 to a low of $15 \%$ in 2013, indicating a greater reduction at beat level than policing area level.

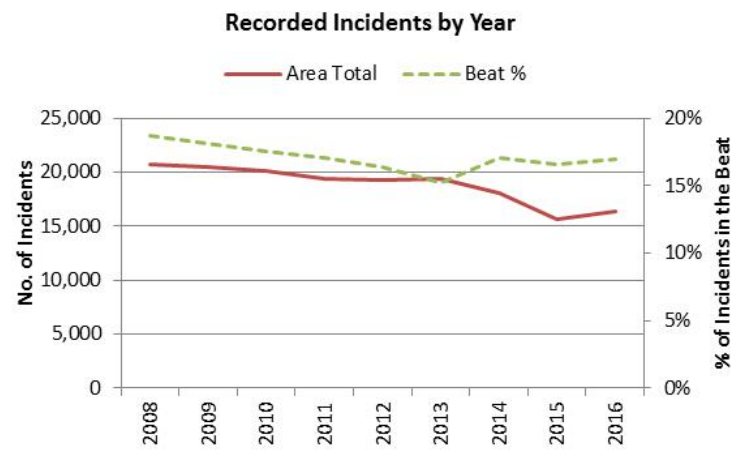

Figure 5. Incident comparison

Other analysis in terms of breaking the incidents down has also been carried out. The number of public safety incidents experienced a slow decline between 2008 and 2014, however there was a sharp decrease in 2015 which could warrant further investigation. The number of anti-social behaviour (ASB) incidents decreased by nearly two thirds over the period, which may have been due to recording changes.

\subsection{Analysis by Hour}

\subsubsection{Method}

In order to analyse the distribution of demand, incidents and crimes were categorized based on the time of day they occurred. The hourly plots are generated by using police data collected for a beat. The data are then aggregated by hour of a day. The advantage of using aggregated data is that the effects of minor observation errors are reduced and the data binning allows quick exploration of the data. The police data also includes some variation due to special events such as concerts, football matches etc. that could cause unusual spikes in particular crime types. Before the aggregation, the raw data are cleaned to remove three types of abnormal records, duplicate records, records with obvious errors (including data entry errors) and the removal of abnormal records from special events to provide the distributions for normal days. 


\subsubsection{Typical examples for crimes}

To understand the time of occurrence of difference crimes distributions per hour can be calculated. Figure 6 illustrates a comparison between the regional level (top) and beat level (bottom) for one example crime type (this can be repeated for other crime types). Depending on the crime type, the exact time of the crime may not be known. In this illustration, this is true for vehicle-related theft crimes where the time of detection by the owner may not be the time of occurrence of the crime. Therefore the earliest and latest possible times are recorded (shown as the lighter and darker histograms respectively). Though the expected peaks are shown for this crime when people come home from work or rise in the morning, slightly different patterns are observed with there being a higher possibility of daytime vehicle crimes in the Beat than the regional level.
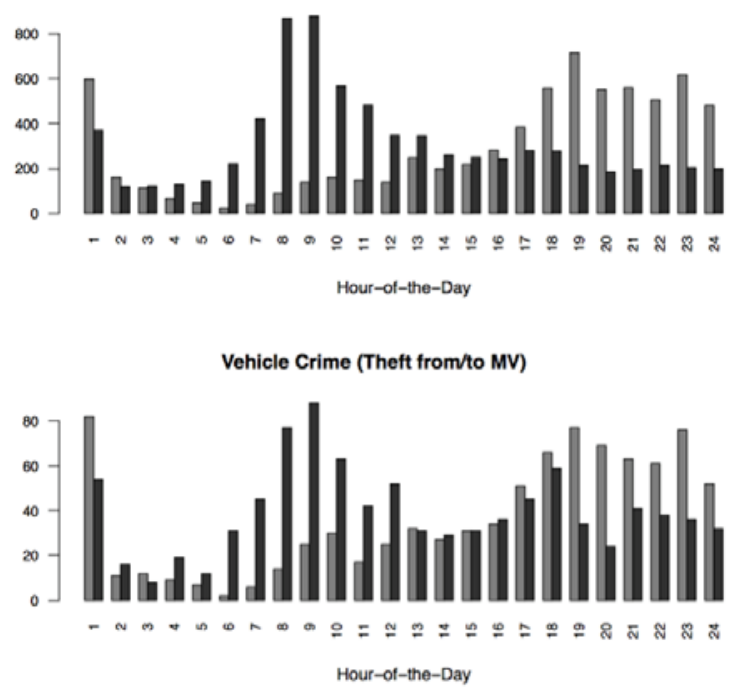

Figure 6. Time spread of crime

\subsubsection{Typical examples for incidents}

Incident records only state the time of the incident being reported to the police. As no further clarification is provided on the actual incident time, the reporting time has been used as an approximation about when the incident happened. The plot of hour-by day of-theweek distribution for overall incidents is shown in Figure 7 , with the top referring to the region, the bottom the beat. The common feature in both for all days of the week is that fewer incidents happen in the early morning when most people are asleep. In the beat specific area the day-of-the-week variation is not that significant.

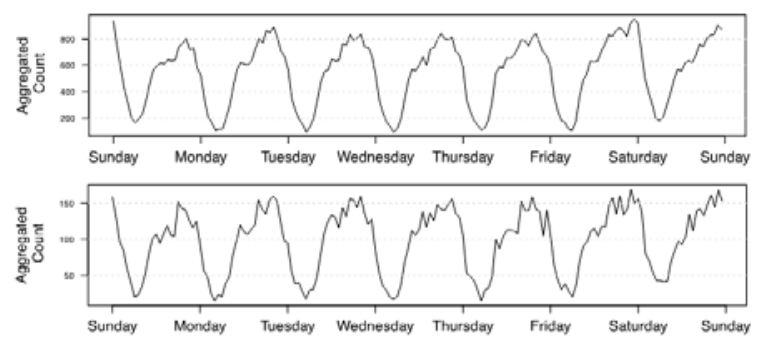

Figure 7. Incident distribution

\subsection{KDE Analysis}

\subsubsection{Method}

Out of the many methods of hotspot identification, Kernel Density Estimation has been adopted as it has been proven to be the method with the best predictive abilities [13]. This method of analysis reduces the effect of boundaries on the results by taking into account crimes in the surrounding area. KDE is performed using a grid overlaid on top of the street map and crime data. The effect of the grid is reduced, for each grid cell, by considering the crimes within a circle whose centre is at the cell centre and whose diameter (bandwidth) is larger than the grid cell size. All the crimes within this bandwidth are assumed to contribute to the intensity of crimes within the cell. The bandwidth radius used for this study is 0.001 degrees, which equates to about $110 \mathrm{~m}^{2}$. The grid cell size and bandwidth have been determined through considering the area of interest and testing the effects of using different sizes. The decision is made based on a compromise between accurate identification of hotspot and computational time. The KDE calculation is then performed on each of the grid cells using Equation 1 [14]. This equation finds the intensity of crimes $\left(\hat{\lambda}_{\tau}(s)\right.$ ) within the bandwidth radius $(\tau)$ as a function of the distance from the cell centre (S). $d_{i}$ is the distance between the grid centre (S) and the point being investigated $S_{i}$. The further the crime from the centre of the grid cell the less it adds to the intensity. Once the crime intensity in the region is known the hotspots are identified as the areas with the highest $n \%$ intensity, where $\mathrm{n}$ is dependent on the situation.

$$
\hat{\lambda}_{\tau}(s)=\sum_{d_{i} \leq \tau} \frac{3}{\pi \tau^{2}}\left(1-\frac{d_{i}^{2}}{\tau^{2}}\right)^{2}
$$




\subsubsection{Results}

Application of the KDE analysis been performed at a regional level. Though this is often done for current data, comparisons over a time period are typically not performed. Doing this analysis has shown migration and emergence of hotspots over time (206-2016). This is illustrated in Figure 8, where (a) shows a hotspot region almost centre screen (the redder area), where as in 2010 (Figure 8b) shows the emergence of a new hotspot. Further analysis of crime type and area can provide more insight into this occurrence.

Following a similar pattern, the Beat level analysis can hone in on specific regions of attention and their migration/emergence. Analysis showed that between 2009 and 2010, a marked difference in the areas affected by crime were identified, with a migration north, with a spot intensified in the north western area. In 2013 the mapping showed similar areas affected but with much lower density, suggesting a significant decrease in crime rates across the entire area. Also in 2014 a northern deviation of crime hotspot is experienced, which further analytics planned to investigate.

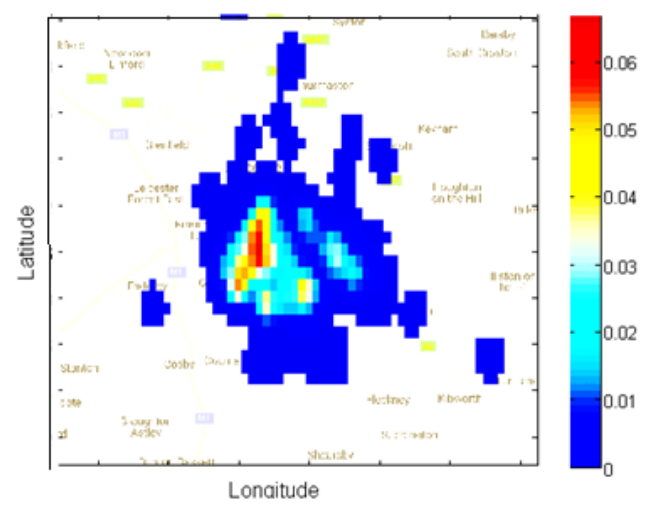

(a) 2007

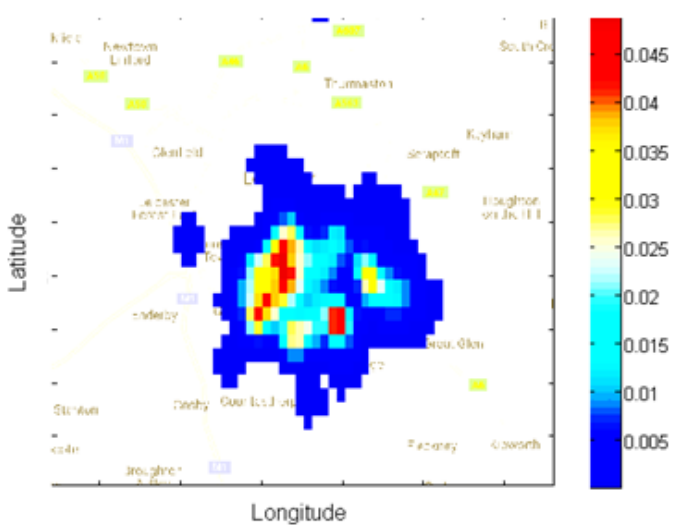

(b) 2010

Figure 8: Regional KDE maps

\section{Drill down analysis}

\subsection{Method of SPC}

Statistical Process Control (SPC) charts are generally used to help with continuous process improvements. In this case, SPC charts were fitted retrospectively to identify trends and highlight occasions where there was a real change to the data. This method reduces knee jerk reactions to changes in data and helps inform the wider picture. The charts using crime data are based on crime start date and those for incidents use incident reported date to plot the data, and each chart covered a year. Upper and lower limits are calculated by Mean+/three standard deviations ( $\mathrm{x}$-bar $\pm 3 \sigma$ ). However as there cannot be minus crime, if the lower control limit was to go below zero, it would be automatically set to zero. The initial limits and average are based on the first twenty points of data. Although there are many suggested rules as to when a process is 'out of control', for this analysis four main rules were considered: (i) Points that fall outside of one of the control limits; (ii) A run of points above or below average; (iii) A run of points all increasing or decreasing; and (iv) The proportion of points within the middle $1 / 3$ of the region between the control limits differs from $2 / 3$. Once a chart went out of control, limits were re-calculated from that point on. This enabled the identification of periods when the frequency of crimes changed and give focus to further drill down analysis.

\subsection{Findings}

Multiple SPCs have been determined and analysed. The findings are illustrated with one year as an example. Figure 9 shows daily recorded crime over a one year period for the beat. Highlighted points represent trends of interest or where the process has "gone out of control". There was a slight increase in the number of recorded crimes between March and April, however, later in the year there are three periods that break the upper control limit.

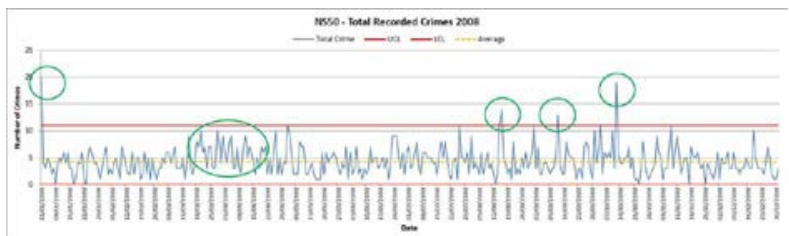

Figure 9. Yearly crime plot 


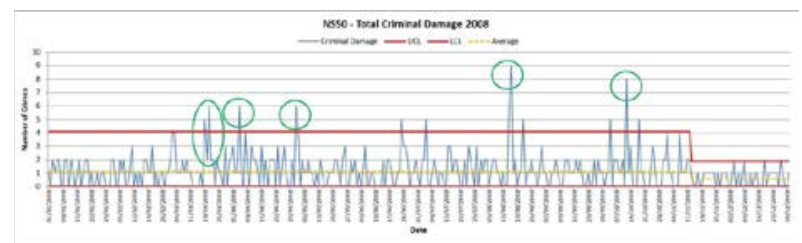

Figure 10. Specific crime type plot

Looking at the SPC chart (Figure 10, representing a specific crime type) for daily criminal damage offences shows a smiliar picture, with points above the upper control limit in March and April, contributing the the overall increase, and peaks later in the year, again contributing to overall peaks. A similar trend of peaks in March and April have been exhibited for other crime types, though with accompanying reductions in May and July. The general increase in offences between March and April, and peaks later in the year is also supported when looking at number of offences by month for the beat, which shows March and October as the highest months (Figure 11).

Looking at the average daily number of recorded crimes for the month of March across the years also revealed that it was the highest in 2008, with an average of over 5 offences a day recorded (an extract from all years is shown in Figure 12 depicting the time window of this increase). This is also higher than the average daily number of crimes for the year, suggesting further analysis into March 2008 is needed to see if there are predictors that can be ascertained to enable future peaks to be identified. Also comparison of years can show patterns of increases/decreases, as illustrated in Figure 13.

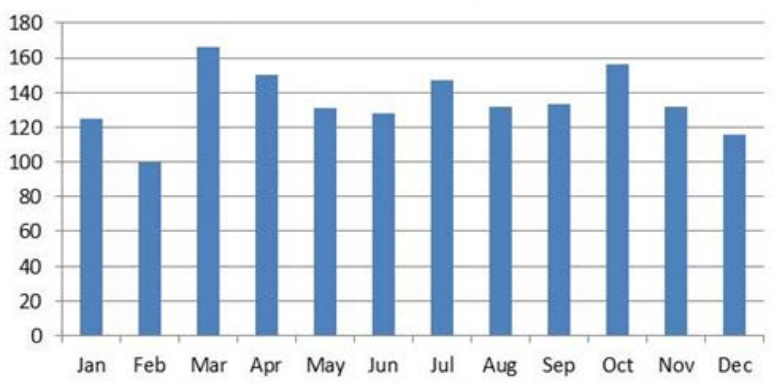

Figure 11: Crimes by month

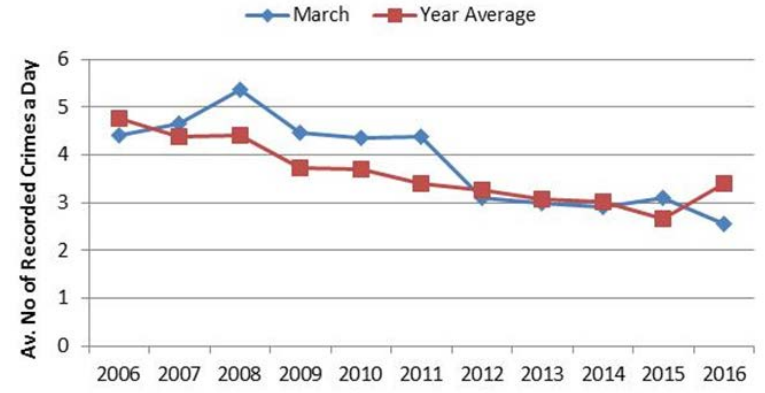

Figure 12: Crimes By year

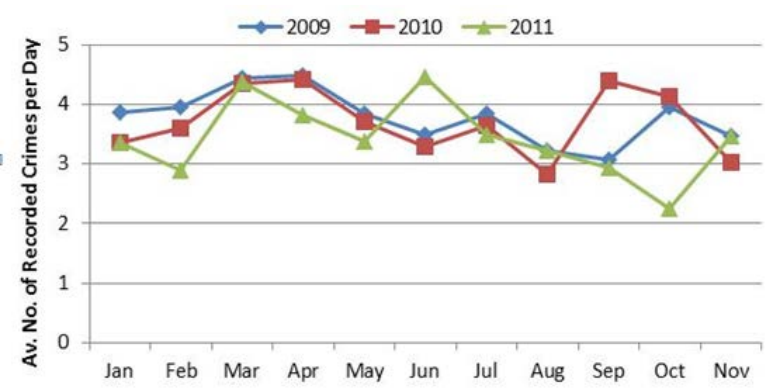

Figure 13: Yearly comparison

\section{Comparison and Strategy implications}

Not only has the analysis enabled a greater understanding of demand profiles for different crime types, different times of the day and for different years for the region and beats, it has provided useful insight into where variations exist. Key findings are: i) crimes in the beat have decreased at a similar rate to the area as a whole (accounting for around 19\% of crime in the area over this time); ii) for this beat, the main crime types are criminal damage and violence (with and without injury), and these occur as a higher proportion of area crime than the overall rate (of 19\%) compared to burglary which is a lower proportion (10\%) indicating differing beat priorities to the region; and iii) identification of changing hotspot areas within the beat influencing beat management.

The trend analysis has shown implications with regards to priority settings. Currently these are devised at a regional level, however this analysis has shown that there can be beat level differences with higher or lower proportions observed. Depending on the priority a different tactic may be required, i.e. Burglaries versus Assault requires differing approaches.

Also it has been noted that proportions of crimes can vary which could have implications with regards to operating mobile Beat teams, such that other beats experiencing higher levels of crime at particular times 
could utilize officers from beats with lower proportions of crime at the same time.

Given that times of crimes can vary too at beat level, this has implications on the information given to inform officers of what to be looking out for - i.e. car thefts common place between a set period of the day, not just at night (which more conforms to the regional picture).

This level of detail for crime and incident analysis has not previously been available to beat commanders. These suggested implications were supported via end user feedback sessions. In these sessions it was revealed that NPA commanders felt they gained a greater level of understanding regarding Beat specific crime and felt that these findings, along with future planned research, can have impact in a number of ways. One is the influence on patrol strategy for neighbourhood officers, e.g. where to patrol to have maximised benefit (to a very low level such as which streets), when, and what types of specific crime are being targeted, therefore informing which tactics to use. Secondly comments were made that the research has the potential to address questions like 'Where does crime prevention advice need to be targeted?' It was seen that the future combination of demographic data could inform media campaigns to specifically target the correct communities in the way that they consume information, e.g. some are more receptive to radio, versus some who are more receptive to social media. Thirdly reference was made that if the work was more widespread it could help make an assessment of the balance of staffing levels on the various neighbourhoods, i.e. are some understaffed given their demand compared to other neighbourhoods? Also such knowledge can also be used to inform supporting activities such as officer training, for example, avoiding peak periods for training would be advisable. Lastly comments were made that if the analysis was to show that particular neighbourhoods have similar issues, or have had similar issues in the past it could ensure that the correct teams are communicating to ensure lessons from the past are learnt and share.

\section{Conclusions}

This research, using data analytics on a large dormant data set, has facilitated a historical investigation to understand changing crime patterns, both at a regional and Beat level, with insights gained which were previously unknown, thus demonstrating the leverage of big data to improve police operations.

Though what might be deemed analytics at a basic level the findings and knowledge elicited has already demonstrated the benefits of such techniques at a macro and micro level, and in addition illustrated differences at these levels for the Police Force. End user feedback has supported the reasoning that this knowledge discovery can have direct implications to operational strategies (with regards to patrolling and communication mechanisms), implications for targeted crime prevention advice and the setting of priority areas at a micro level, and to service provision (including staffing level balance). Future work will investigate wider beat level comparison, practical implications of differing operational strategies in more detail and end-user potential impact avenues. This is planned to be coupled with inclusion of tools for improved visual analytics to provide opportunities to directly engage higher management and facilitate further exploration of data for guided analysis with the use of more advanced analytics methods for future predictions.

\section{References}

[1] G. Cao, Y. Duan \& G. Li. Linking Business Analytics to Decision Making Effectiveness: A Path Model Analysis. IEEE Transactions on Engineering Management, 62(3):384-395, 2015.

[2] R. Kohavi, N. J. Rothleder \& E. Simoudis. Emerging trends in business analytics. Communications of the ACM, 45(8):345-348, 2002.

[3] S. Tyagi. Using data analysis for greater profits. Journal of Business Strategy, 24(3):12-14, 2003.

[4] J. Car, W. S. Tan, Z. Huang, P. Sloot \& B. D. Franklin. eHealth in the future of the medications management: personalization, monitoring and adherence. BMC Medicine, 15(1):73, 2017.

[5] M. L. Stone. Big Data for Media. Report from the Reuters Institute for the Study of Journalism, (1):1-5, 2014.

[6] Malik, A., Maciejewski, R., Towers, S., McCullough, S., Ebert, D.S. Proactive spatiotemporal resource allocation and predictive visual analytics for community policing and law enforcement. IEEE

Transactions on Visualization and Computer Graphics, 20(12), 1863-1872, 2014.

[7] Leigh, J., Dunnett, S., \& Jackson, L. Predictive police patrolling to target hotspots and cover response demand. Annals of Operations Research, 1-16, 2017.

[8] Godwin, A., Stasko, J. HotSketch: Drawing police patrol routes among spatiotemporal crime hotspots, Proceedings of the 50th Hawaii International Conference on System Sciences, HICSS 2017, 1372-1380, Hilton Waikoloa Village, USA, 2017. 
[9] W. L. Perry, B. McInnis, C. C. Price, S. C. Smith \& J. S. Hollywood. Predictive Policing: The Role of Crime Forecasting in Law Enforcement Operations. Santa Monica, CA: RAND Corporation, (1):8-15, 2013.

[10] Bruin, J.S.D., Cocx, T.K., Kosters, W.A., Laros, J.F.J., Kok, J.N. Data mining approaches to criminal career analysis. Proceedings of the 6th International Conference on Data Mining, ICDM 2006, 171-177, Washington, DC, USA, 2006.

[11] Chen, H., Chung, W., Xu, J.J., Wang, G., Qin, Y., Chau, M. Crime data mining: a general framework and some examples, Computer, 37(4), 50-56, 2004.

[12] Yu, C.H., Ward, M.W., Morabito, M., Ding, W. Crime forecasting using data mining techniques. Proceedings of the 11th IEEE International Conference on Data Mining Workshops, ICDMW 2011, 779-786, Washington DC, USA, 2011.
[13] S. Chainey, L. Tompson and S. Uhlig, "The utility of hotspot mapping for predicting spatial patterns of crime," in Security Journal, Palgrave Macmillan. 2008, vol. 21, pp. 4-28.

[14] A. Gatrell, T. Bailey, P. Diggle and B. Rowlingson, "Spatial point pattern analysis and its applications in geographical epidemiology," in Transactions of the Institute of British Geographers, 1996, vol. 21, no. 1, pp. 256-274. 